\title{
Useful Clinical Features for the Selection of Ideal Patients with Atrial Fibrillation for Mapping and Catheter Ablation
}

\author{
Niraj Mehta, Maria Zildany Pinheiro Távora, Noriaki Takeschita, Edilberto Figueiredo, \\ Ricardo M. Lourenço, Hélio Germiniani, Dalton Précoma \\ Curitiba, PR - Brazil
}

\begin{abstract}
Objective - To identify useful clinical characteristics for selecting patients eligible for mapping and ablation of atrial fibrillation.
\end{abstract}

Methods - We studied 9 patients with atrial fibrillation, without structural heart disease, associated with: 1) antiarrhythmic drugs, 2) symptoms of low cardiac output, and 3 ) intention to treat. Seven patients had paroxysmal atrial fibrillation and 2 had recurrent atrial fibrillation.

Results - In the 6 patients who underwent mapping (all had paroxysmal atrial fibrillation), catheter ablation was successfully carried out in superior pulmonary veins in 5 patients (the first 3 in the left superior pulmonary vein and the last 2 in the right superior pulmonary vein). One patient experienced a recurrence of atrial fibrillation after 10 days. We observed that patients who had short episodes of atrial fibrillation on 24-hour Holter monitoring before the procedure were those in whom mapping the focus of tachycardia was possible. Tachycardia was successfully suppressed in 4 of 6 patients. The cause of failure was due to the impossibility of maintaining sinus rhythm long enough for efficient mapping.

Conclusion - Patients experiencing short episodes of atrial fibrillation during 24-hour Holter monitoring were the most eligible for mapping and ablation, with a final success rate of $66 \%$, versus the global success rate of $44 \%$. Patients with persistent atrial fibrillation were not good candidates for focal ablation.

Keywords: atrial fibrillation, radiofrequency catheter ablation, mapping

Hospital Angelina Caron - Campina Grande do Sul, PR

Mailing address: Niraj Mehta - Rua Almirante Tamandaré, 503/52 - 80050-230

Curitiba, PR - Brazil - E-mail: Niraj@netbank.com.br
Atrial fibrillation is the most common supraventricular arrhythmia observed in clinical practice, corresponding to $35 \%$ of hospital admissions due to arrhythmias ${ }^{1}$. This type of arrhythmia leads to changes in the patient's quality of life and is responsible for significant morbidity and mortality ${ }^{2,3}$. Its treatment involves long-term use of antiarrhythmic drugs with only partial control of tachyarrhythmia crises ${ }^{4-7}$.

A major revolution has occurred in the treatment of arrhythmias in this last decade with the arrival of ablation of supraventricular tachyarrhythmia using radiofrequency energy ${ }^{8}$. Patients who depended on antiarrhythmic drugs, which are not always effective, were totally cured after radiofrequency ablation ${ }^{9,10}$. If this technique is used effectively in patients with atrial fibrillation, it may benefit them, even if it may be used in only a portion of patients.

Currently, 2 technical approaches exist for curative catheter ablation of atrial fibrillation: the creation of lines of block simulating the surgery of Cox et al ${ }^{11}$, and the search for ectopic foci responsible for atrial fibrillation ${ }^{12}$.

Several physicians have tested the possibility of creating lines of block to simulate the Cox surgery, and although initially successful when performed by Swartz et al ${ }^{13}$, this technique has proven difficult, complicated, and slow, leading to complications in $31 \%$ of patients. Apart from the significant incidence of complications, the $79 \%$ success rate achieved by Swartz et al has not been reproduced by others, even though they used advanced technology mapping (Carto system) ${ }^{14}$. Possibly, better designed catheters for a more complete and faster creation of lines of block are necessary so that this technique can be feasible and applicable in most electrophysiology study centers.

On the other hand, the search for the focal sources that cause atrial fibrillation and can be cured with ablation is not recent. It is known that patients with Wolff-ParkinsonWhite syndrome have a higher incidence of atrial fibrillation than the rest of the population ${ }^{15}$ and that the elimination of the accessory pathway using ablation techniques also generally suppresses atrial fibrillation. Patients with 
tachycardia caused by nodal reentry have a higher incidence of atrial fibrillation ${ }^{16}$, and the frequency of crises can be reduced after elimination of tachycardia ${ }^{17}$.

Recently, we have observed that in some patients a focus of very rapidly occurring atrial tachycardia can lead to atrial fibrillation. Ablation of atrial tachycardia may lead to definite elimination of atrial fibrillation ${ }^{12}$.

Because patients who present with focal atrial fibrillation clearly benefit from mapping and ablation techniques, selecting ideal candidates is very important. Currently, we do not know the actual incidence of focal atrial tachycardia as the cause of atrial fibrillation. Likewise, we do not yet know how to select ideal candidates. Another remaining question is whether or not this technique can be widely used in all electrophysiological study centers.

This article reports our experience in a limited number of patients with paroxysmal or recurrent atrial fibrillation, who underwent attempts at mapping and focal ablation, taking into account clinical features that may be useful for the selection of the ideal candidate for mapping and ablation.

\section{Methods}

From March 1999 to January 2000,9 male patients with paroxysmal or recurrent atrial fibrillation were referred for electrophysiological study and radiofrequency ablation. The criteria for selecting patients was the presence of paroxysmal or sustained atrial fibrillation, without structural heart disease, related to the following clinical data: 1) refractoriness to conventional antiarrhythmic medications, including amiodarone, 2) presence of symptoms of low output or any other disabling symptom, and 3) intention to treat. All patients underwent 24-hour Holter monitoring and echocardiography. We considered paroxysmal atrial fibrillation as fibrillation that can be spontaneously reverted at least once, and recurrent atrial fibrillation as fibrillation that required electrical cardioversion to be reverted. Clinical features of patients are shown in table I. No patient had structural heart disease on ECG, and all had normal sized left atrium $(<4 \mathrm{~cm})$. In patients with recurrent atrial fibrillation, the period of sustained arrhythmia was less than 6 months in 1 patient and less than 2 years in the other. Initially, all patients but the first (who had rare episodes of atrial fibrillation and whose arrhythmia type was irregular atrial tachycardia) received anticoagulation with dicumarol or warfarin for 3 weeks before the procedure. One week before the procedure, oral anticoagulation medication was discontinued and was replaced by subcutaneous heparin $20 \mathrm{mg}$, up to the day of the electrophysiological study. Antiarrhythmic drugs except for amiodarone were discontinued for a particular period of time. Amiodarone was discontinued for at least a week prior to the procedure.

Electrophysiological procedures were performed in a hemodynamic room with a 16-channel-cardiac-polygraph $\left(\mathrm{TEB}^{\circledR}\right)$. Initially, patients with recurrent atrial fibrillation underwent electrical cardioversion. Patients whose cardioversion was successful and those with sinus rhythm underwent femoral vein puncture to position the diagnostic catheters. In patients that did not have spontaneous atrial extrasystoles, drug stimulation techniques (isoproterenol infusion and adenosine) and electrical stimulation were used to induce atrial fibrillation or atrial extrasystoles. We did not use other provocative maneuvers. A classical transseptal puncture was made, using the Brockenborough technique, and then a $7 \mathrm{~F}$ ablation catheter was manipulated with an electrode distance of $2 \mathrm{~mm}$ (Cordis-Webster ${ }^{\circledR}$ ) through the left atrium. The mapping technique used was the detection of earlier electrical activity during atrial extrasystoles and the recording of double potentials (pulmonary vein potential followed by atrial electrogram). To perform mapping, fluoroscopy was done in the anteroposterior projection, and in some patients the right anterior oblique projection associated with a cranial projection of 15 degrees was used. In the sites of precocious electrical activity, a 15-W radiofrequency current was delivered for up to $15 \mathrm{~s}$ each application. Application was discontinued if the patient complained of pain. Applications were performed in only 1 pulmonary vein. After the procedure, patients were followed-up and put on 24-hour Holter monitoring (performed up to 15 days after the procedure). Procedural success was defined as the definite interruption of tachycardia and the inability to induce it again. Clinical success was the absence of symptoms in a 4.8-month follow-up. Twenty-four-hour Holter monitoring was performed up to 15 days after the procedure.

Antiarrhythmic drugs were discontinued in patients who were successfully treated based on laboratory findings. We administered antiarrhythmic drugs to those patients who did not experience successful ablation. All patients were administered warfarin for up to 3 months after the procedure.

\section{Results}

We observed a global success rate in this study of $44 \%$. However, the final success rate (without recurrence) in the subgroup of patients with paroxysmal nonsustained atrial fibrillation on 24-hour Holter monitoring prior to the procedure was $66 \%$ (4 of 6 patients) (table II). Causes of failure are described in table III. We did not observe complications following the procedure.

Endocardial mapping was possible in 8 of the 9 patients (we could not revert 1 patient's recurrent atrial fibrillation to sinus rhythm despite multiple electrical cardioversions). We performed ablation in 6 of the 8 mapped patients. One patient (number 5) had recurrent atrial fibrillation initially cardioverted to sinus rhythm before puncturing was started. In this patient, after transseptal puncture, mapping was initiated in the presence of several episodes of nonsustained atrial tachycardia. However, after 20 minutes 


\begin{tabular}{|cccccc|}
\hline \multicolumn{5}{c|}{ Table I - Clinical characteristics of patients referred for electrophysiological study } \\
\hline Pts & Age (years) & Sex & Clinical Presentation & Electrophysiological Study indication & Previous Holter \\
\hline 1 & 44 & $\mathrm{M}$ & Paroxysmal AF & Refractoriness to drugs & AF (NSAT-20) \\
2 & 68 & $\mathrm{M}$ & Paroxysmal AF & Low output & AF (NSAT-565) \\
3 & 82 & $\mathrm{M}$ & Paroxysmal AF & Low output & AF (NSAT-16) \\
4 & 48 & $\mathrm{M}$ & Paroxysmal AF & Refractoriness to drugs & AF (NSAT-33) \\
5 & 51 & $\mathrm{M}$ & Recurrent AF & Refractoriness to drugs & AF(afterECV,NSAT) \\
6 & 38 & $\mathrm{M}$ & Paroxysmal AF & Intention to treat & AF (NSAT-2084). \\
7 & 51 & $\mathrm{M}$ & Recurrent AF & Refractoriness to drugs & AF \\
8 & 35 & $\mathrm{M}$ & Paroxysmal AF & Refractoriness to drugs & AF (NSAT-18) \\
9 & 30 & M & Paroxysmal AF & Intention to treat & Rare atrial ectopy \\
\hline
\end{tabular}

M- male; AF- atrial fibrillation; ECV- electrical cardioversion; NSAT- nonsustained atrial tachycardia. The number that follows "NSAT" corresponds to the number of nonsustained atrial tachycardia on 24-hour Holter monitoring.

\begin{tabular}{|lccccc|}
\hline \multicolumn{4}{|c|}{ Table II - Success rate of ablation in patients with short-duration atrial fibrillation (nonsustained atrial tachycardia) on 24-hour Holter monitoring } \\
prior to the procedure compared with the total group of patients
\end{tabular}

\begin{tabular}{|c|c|c|}
\hline \multicolumn{3}{|c|}{ Table III - Causes of failure } \\
\hline Pts & Transeptal puncture & Causes of unsuccessful mapping \\
\hline 4 & Yes & Insufficient atrial ectopy \\
\hline 5 & Yes & $\begin{array}{l}\text { sufficient mapping duration (degeneration } \\
\text { to } \mathrm{AF} \text { nonrespondent to cardioversion) }\end{array}$ \\
\hline 7 & No & $\begin{array}{c}\text { (AF nonresponsive to cardioversion) } \\
\text { Mapping not performed }\end{array}$ \\
\hline 9 & Yes- & $\begin{array}{l}\text { Insufficient atrial ectopy } \\
\text { (application unsuccessful) }\end{array}$ \\
\hline
\end{tabular}

of mapping, sustained atrial fibrillation occurred, which was not reverted, not even after the use of 5 shocks of 360J. In another patient (number 4), radiofrequency was not applied because the patient did not have ectopy, making it impossible for mapping to be performed.
Two of the 3 patients in whom proper mapping was achieved and radiofrequency was applied had spontaneous induction of atrial fibrillation provoked by ectopic beats (fig. 1). In the sites selected for ablation, earlier electrical activity occurred in the left superior pulmonary vein in 4 patients and in the right superior pulmonary vein in 2 patients (table IV). Laboratory success was achieved in 5 patients: 4 are free of preexcitation, atrioventricular reentry tachycardia, and we could not induce atrial fibrillation in patient number eight. Laboratory success was not achieved in patient number 9 due to rare atrial ectopy, which made it, impossible to institute precise mapping. Radiofrequency was applied at sites with precocious electrograms and in the presence of double potentials (pulmonary vein potential and atrial electrogram). Three successful electrograms with these features were observed (figs. 2, 3, 4 and 5). In 2 cases, ablation was performed inside the pulmonary vein (fig. 6), and in the

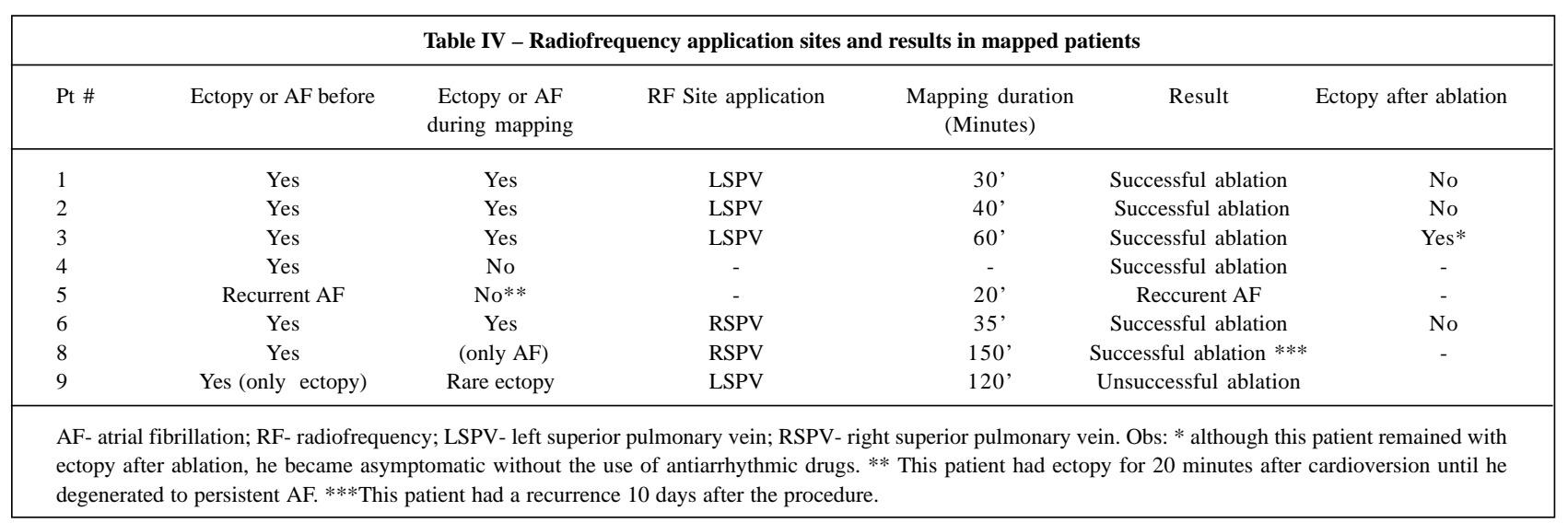




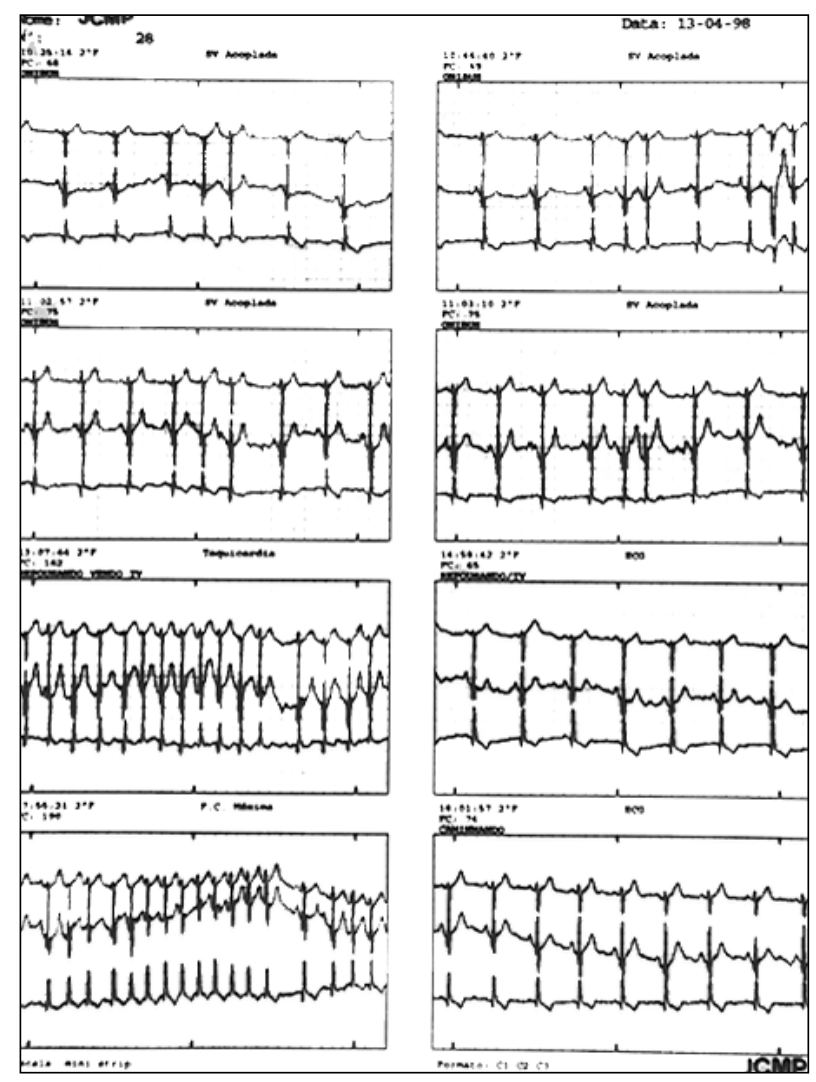

Fig. 1 - Records of 24-hour Holter monitoring with several electrocardiographic tracings. Several episodes of short-standing atrial fibrillation were observed (in fact, they were nonsustained atrial tachycardia). They were mainly seen in the last 2 tracings of the first column.

other 3 ablation was applied in the pulmonary vein ostia with low precocity related to the $\mathrm{P}$ wave (fig. 3 ). When ablation was applied inside the pulmonary vein, precocity related to the $P$ wave $(118 \mathrm{~ms}$ and $70 \mathrm{~ms}$ ) was more evident when compared with ablation applied to the pulmonary vein ostia (28ms, $48 \mathrm{~ms}$, and $36 \mathrm{~ms}$ ).

Clinical success (absence of symptoms) was obtained in 4 patients (numbers 1,2,3, and 6), in an average 4.8-month follow-up. On 24-hour Holter monitoring, we also observed the elimination of nonsustained atrial tachycardia (numbers $1,2,6)$. One patient who achieved laboratory success (noninduction of atrial fibrillation) experienced recurrence of atrial fibrillation 10 days after the procedure (number 8 ).

In all patients who achieved laboratory success (number 1,2,3,6, and 8), antiarrhythmic drugs were discontinued so that clinical results and possible recurrences could be observed. In patients who did not achieve laboratory success (number 4, 5, 7, and 9) or in whom clinical recurrences occurred (number 8 ), antiarrhythmic drugs were reintroduced.

Twenty-four hour Holter monitoring preablation and postablation (table $\mathrm{V}$ ) reveals an interesting finding. The presence of frequent episodes of nonsustained atrial tachycardia on 24-hour Holter preablation was associated with a greater rate of success.

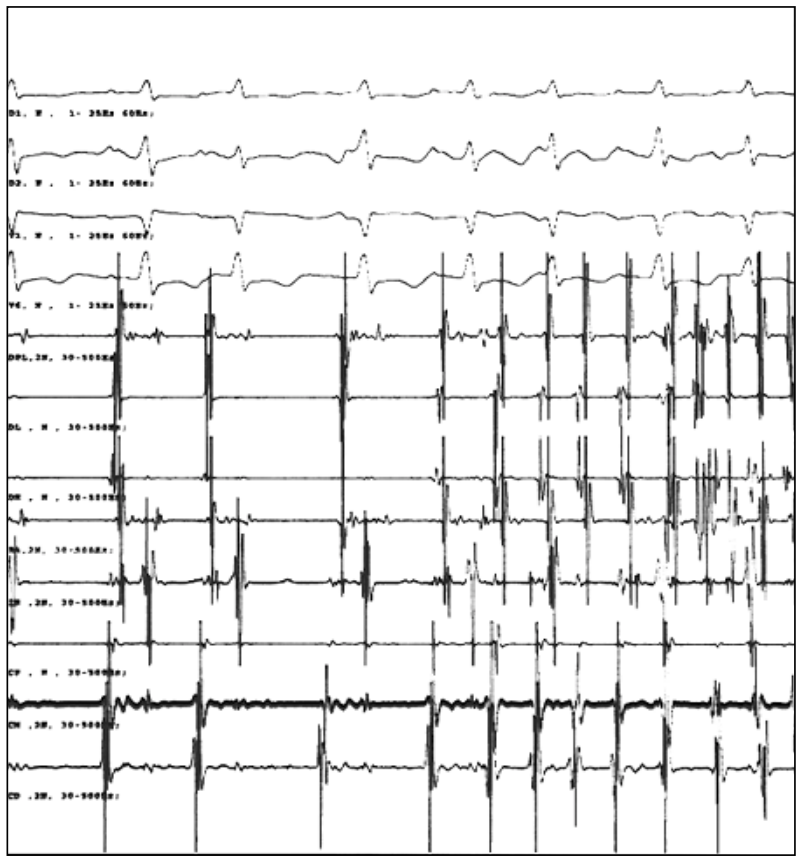

Fig. 2-Record of surface derivations I, II, $\mathrm{V}_{1}$ and $\mathrm{V}_{6}$ and of intracavity electrograms of the right atrium (ADPL, ADL, ADH, IE4), His bundle (HIS), and left atrium (SCP, SCM, SCD) of spontaneous induction of atrial fibrillation initiated by atrial extrasystoles. It was observed that the fifth QRS is preceded by an atrial extrasystole (ectopic $\mathrm{P}$ wave observed in DII), followed by a rapid sequence of atrial extrasystoles with induction of atrial fibrillation on the surface electrocardiogram and maintenance of intracavity extrasystole activation.

\section{Discussion}

Catheter ablation of atrial fibrillation is currently one of the greatest challenges in electrophysiology. This arrhythmia is difficult to control with antiarrhythmic drugs and has the potential of becoming permanent as the number and duration of episodes increase. The possibility of cure, even if only in a small number of patients, is extremely rewarding,

\begin{tabular}{|c|c|c|c|}
\hline \multicolumn{4}{|c|}{$\begin{array}{l}\text { Table V - Findings of } 24 \text {-hour Holter monitoring before and after } \\
\text { ablation of patients referred for electrophysiological study }\end{array}$} \\
\hline Pts & Previous Holter & Ablation result & Holter after \\
\hline 1 & AF (NSAF-20) & Successful ablation & Absence of NSAT \\
\hline 2 & AF (NSAF-565) & Successful ablation & Absence of NSAT \\
\hline 3 & AF (NSAF-16) & Successful ablation & $\begin{array}{c}\text { Presence of NSAT } \\
(8)^{* *}\end{array}$ \\
\hline 4 & AF (NSAF-33) & Successful ablation & Absence of NSAT \\
\hline 5 & $\begin{array}{c}\mathrm{AF}(\text { after } \\
\mathrm{ECV}, \mathrm{NSAT})\end{array}$ & Persistent AF & Persistent AF \\
\hline 6 & AF (NSAT-2084). & Successful ablation & Absence of NSAT \\
\hline 7 & $\mathrm{AF}$ & Persistent AF & Persistent AF \\
\hline 8 & AF (NSAT-18) & Successful ablation $*$ & $\begin{array}{l}\text { Paroxysmal AF } \\
\text { (6-hour duration) }\end{array}$ \\
\hline 9 & Rare atrial ectopy & Unsuccessful ablation & Rare atrial ectopy \\
\hline \multicolumn{4}{|c|}{$\begin{array}{l}\text { AF- atrial fibrillation; ECV- electrical cardioversion; NSAT- nonsustained } \\
\text { atrial tachycardia. The number that follows "NSAT" corresponds to the } \\
\text { number of episodes of nonsustained atrial tachycardia on } 24 \text {-hour Holter } \\
\text { monitoring. * This patient had recurrence } 10 \text { days after the procedure. ** } \\
\text { This patient remained with episodes of nonsustained atrial tachycardia on } \\
\text { 24-hour Holter monitoring, but clinically he was asymptomatic. }\end{array}$} \\
\hline
\end{tabular}




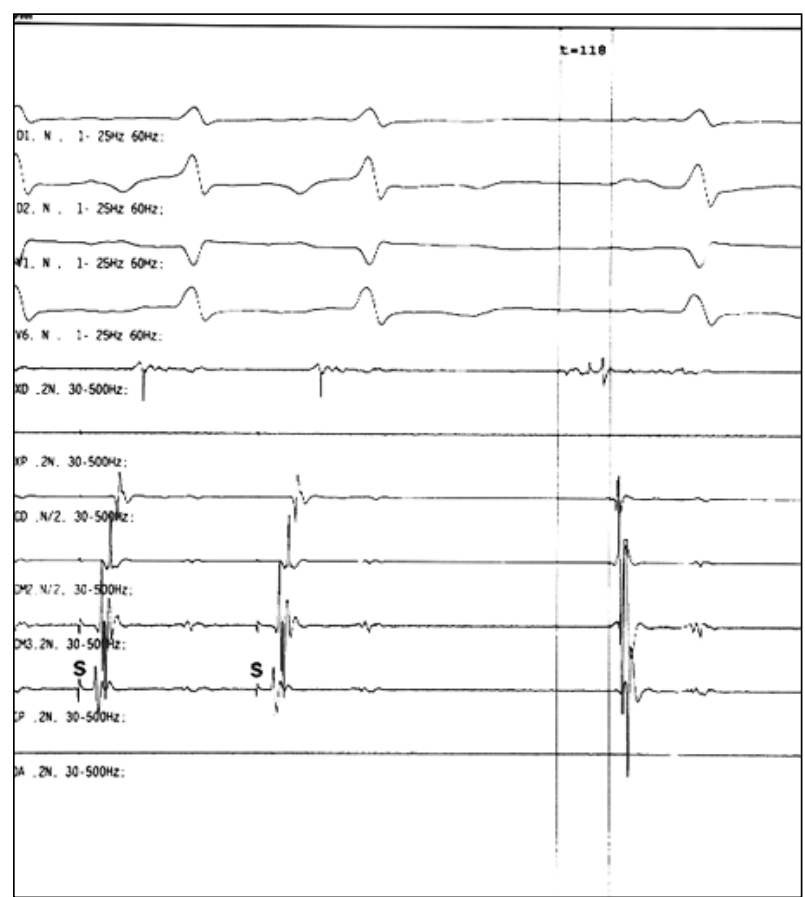

Fig. 3-Record of surface derivations I, II, $\mathrm{V}_{1}$ and $\mathrm{V}_{6}$ and of intracavity electrograms of catheter ablation by double distal electrodes (EXD) and by the double of the proximal electrode (EXP), of the left atrium (SCD, SCM2, SCM3, SCP) and of the right atrium (ADA) of the application site of successful radiofrequency. In the first intracavity electrogram of the catheter ablation (EXD), a $118 \mathrm{~ms}$ precocious ectopic beat was observed in relation to the surface $P$ wave.

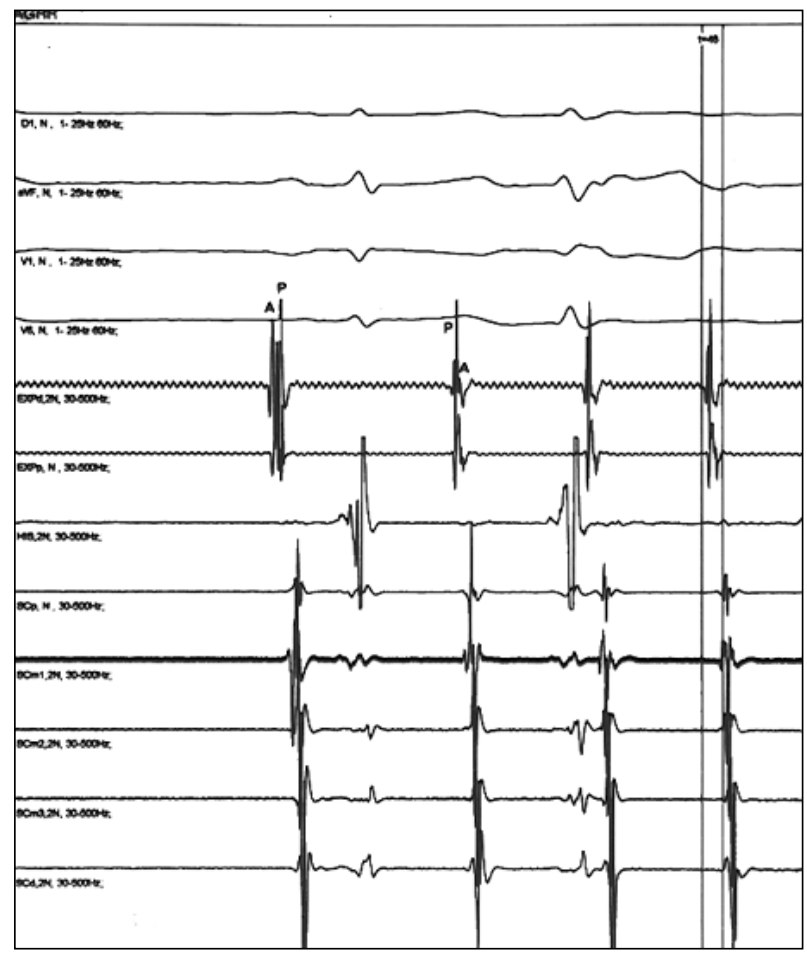

Fig. 4-Record of surface derivations $\mathrm{I}$, toVF, $\mathrm{V}_{1}$ and $\mathrm{V}_{6}$ and of intracavity electrograms of the ablation catheter by the double distal electrode (EXD) and by the double proximal electrode (EXP), of His bundle (HIS), and of the left atrium (SCP, SCm1, $\mathrm{SCm}, \mathrm{SCm} 3, \mathrm{SCD}$ ) of the application site of successful radiofrequency. In the first intracavity electrogram of the ablation catheter (EXD), we can observe that the first beat is sinusal (with the pulmonary vein potential following the atrial electrogram). In the following beats, we can observe fusion and inversion of electrograms (atrium potential follows the potential of pulmonary vein).

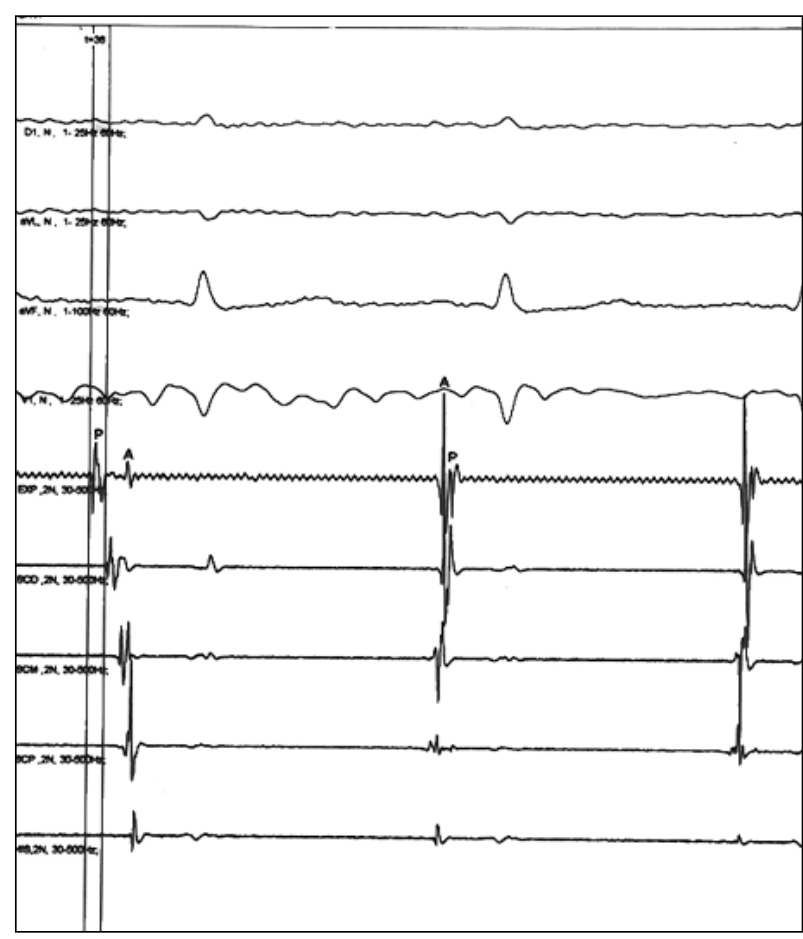

Fig. 5 - Record of surface derivations I, VL, VF, and $\mathrm{V}_{1}$ and of intracavity electrograms of catheter ablation by the distal electrode pair (EXP), of the left atrium (SCD, SCM, SCP) and of the His bundle (HIS) of the successful application site of radiofrequency. In this electrogram, we observe that the first beat is an atrial ectopy (with a left atrium potential following the electrogram of a $36 \mathrm{~ms}$ precocious pulmonary vein in relation to the distal coronary sinus electrogram-SCD). In the following sinusal beats, we observed electrogram inversion (pulmonary vein potential follows left atrium potential).

due to the significant morbidity and mortality associated with this frequent disease.

Criteria for selecting patients for mapping and catheter ablation have not been appropriately defined. Because this ablation technique has only just recently begun to be used for atrial fibrillation, we do not know exactly which patients benefit most from the procedure. Although this curative technique is highly desirable for all patients, the procedure is complex, involves risks, and the reproducibility success rate has not been totally proved. For this reason, it is important to identify the patient with the greatest chance of a cure with the ablation procedure.

Atrial fibrillation focal ablation was first described by Haïssaguerre et al ${ }^{18}$, and its use is considered a great achievement because it was believed that atrial fibrillation could never be suppressed by a focal application. In another study with 9 patients carried out by the same group of researchers, the most relevant clinical data reported for identifying patients eligible for the ablation technique is the finding of atrial fibrillation associated with episodes of irregular monomorphic tachycardia or atrial extrasystoles without structural heart disease ${ }^{12}$.

Strict selection criteria were used by the same authors in another study. Only patients with atrial fibrillation resistant to at least 2 drugs, having at least 1 episode of atrial fibrillation every 2 days, and, having frequent isolated atrial ectopic beats (more than $700 / \mathrm{h}$ ) were included ${ }^{19}$. In a study 


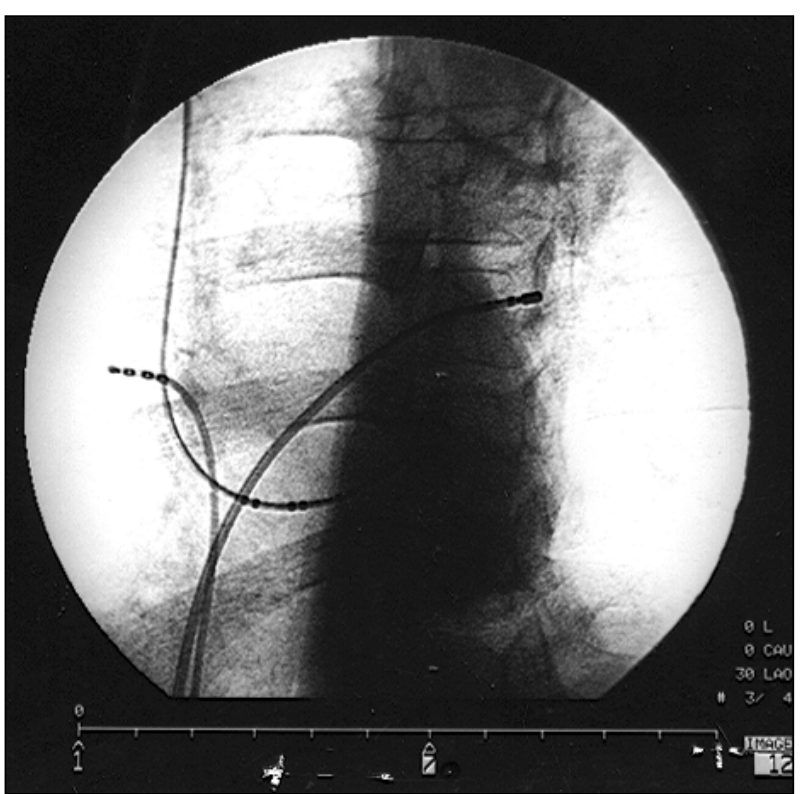

Fig. 6 -A left anterior oblique cine frame at a $30^{\circ}$ position of the ablation catheter at the successful application site. We observed that the ablation catheter is inside the superior left pulmonary vein placed through a transeptal puncture.

by Arentz et al, ${ }^{20}$ in 232 patients with paroxysmal atrial fibrillation to on 24-hour Holter monitoring who underwent ablation, only 23 patients with atrial tachycardia or frequent monomorphic atrial extrasystoles were selected. Of those, 10 had frequent paroxysmal atrial fibrillation (more than 2 episodes per week) and were resistant to more than 1 antiarrhythmic drug. In this subgroup, it was possible to identify a focal source in only 3 patients who successfully underwent ablation.

These extremely rigorous criteria for attempts at mapping and ablation were quickly changed. Interesting observations arose from studies of chronic atrial fibrillation, where the attempt at eliminating arrhythmia was based on the performance of linear lesions simulating Cox surgery. In these studies, the organization of atrial activity either shortened or prevented episodes of atrial fibrillation, suggesting focal activity with atrial extrasystoles, which are the main triggering mechanisms of atrial fibrillation episodes before ablation. These atrial ectopies were identified in at least $40 \%$ of patients and had not been obvious before the procedure due to long-lasting atrial fibrillation. We also observed that the pulmonary veins are the main source of these ectopic foci ${ }^{20-22}$. It is still unknown whether in these patients with chronic atrial fibrillation ablation of these triggering foci prior to linear ablation is enough to suppress arrhythmia ${ }^{23}$. These impressive findings raised expectations that those patients with long- lasting atrial fibrillation could also be eligible for mapping and ablation. This belief encouraged the inclusion of an increasing number of patients with long-standing paroxysmal fibrillation or recurrent atrial fibrillation. Lau et $\mathrm{al}^{24}$ selected for mapping and ablation not only patients with paroxysmal atrial fibrillation but also patients with chronic internal defibrilla- tion. In this study, $20 \%$ of patients with chronic atrial fibrillation had spontaneous atrial fibrillation reentry, which was used as a guide for mapping and ablation. Todd et $\mathrm{al}^{25}$ demonstrated that focal atrial arrhythmias commonly occur after electrical cardioversion in patients with recurrent atrial fibrillation. In this study, $39 \%$ of patients (14 of 36) had focal atrial tachycardias that were mapped. This finding was very important because it increased confidence in the possibility that patients with chronic atrial fibrillation could be included for mapping and ablation. Takahashi et al ${ }^{26}$ performed successful ablation in 3 of 6 patients with recurrent atrial fibrillation. Mapping was not successful in 2 patients ( 1 due to the absence of ectopy and 1 due to the presence of multiple foci).

Structural heart disease is another factor that excludes patients for mapping. Structural heart disease implies greater electrical disarrangement and fewer chances of focal ablation. However, in clinical studies, data are controversial. In the study by Todd et $\mathrm{al}^{25}$, the absence of structural heart disease was the only independent predictor of a focal source of atrial fibrillation (12 of 24 patients without structural disease had focal atrial tachycardia). Although the presence of structural heart disease seems to clearly hinder successful ablation, Vergara et $\mathrm{al}^{27}$, when analyzing clinical predictors of successful atrial fibrillation ablation, observed that structural heart disease was not associated with fewer chances for ablation. In this study, a greater number of atrial fibrillation foci in the same patient and the presence of left atrial flutter were associated with a greater incidence of recurrence, reflecting a greater extension of atrial disease.

The presence of structural heart disease is no longer a contraindication for mapping and ablation of focal atrial fibrillation in a series of patients studied by the group of French researchers, previously mentioned ${ }^{28}$. In the last 10 months of the study, this group ${ }^{29}$ used as a criterion for inclusion of patients for mapping and focal ablation of resistant atrial fibrillation. This fast and progressive evolution as an indication for mapping and ablation in patients for identifying discreet foci as the source of atrial fibrillation caused difficulty for the clinical cardiologist in the selection and referral of patients for electrophysiological study.

In our limited series of patients, we observed that mapping of pulmonary veins to search for atrial tachycardia foci that cause atrial fibrillation is feasible, although the selection of the ideal patient for the procedure is of utmost importance to achieve the best results.

In the present study, criteria for inclusion were comprehensive (including patients with paroxysmal and recurrent atrial fibrillation). Our results indicate that patients with short- standing atrial fibrillation (irregular atrial tachycardia simulating atrial fibrillation) on 24-hour Holter monitoring were those with the highest success rate. Because they were in sinus rhythm and had atrial paroxysm, a precise localized mapping of the source of these foci was possible, revealing that those patients were the best candidates. On the other hand, it was more difficult to map 
patients with recurrent atrial fibrillation, because they had sinus rhythm with frequent atrial extrasystoles of the same morphology.

Causes of failure in our series were mainly rare atrial ectopy and atrial fibrillation resistant to electrical cardioversion. In 1 patient (number 9), atrial ectopy was very rare during the procedure, hindering precise mapping. In another case (number 4), the rarity of ectopic episodes hindered mapping, not allowing the selection of the focus for application of radiofrequency. In patient number 8 , we were able to perform mapping and ablation of a focus in the right superior pulmonary vein, despite infrequent ectopy during mapping. We considered noninducibility of atrial fibrillation as a laboratory success. This patient, however, had recurrence of atrial fibrillation 10 days after the procedure. In late follow-up, this patient had significant clinical improvement in symptoms without the use of antiarrhythmic medication.

We were not able to perform focal mapping in the 2 patients (number 5 and 7) with recurrent atrial fibrillation. In one, conversion of atrial fibrillation to sinus rhythm (number 7) was possible. He was a former smoker of 60 cigarettes a day and had a dilated anteroposterior thoracic diameter, in which, even cardioversion techniques with 2 simultaneous charges were unsuccessful. In the other patient, atrial fibrillation was converted, but episodes of nonsustained atrial tachycardia degenerated to atrial fibrillation in a short period of time and were refractory to cardioversion. Internal defibrillation may be a useful technique for converting atrial fibrillation in these patients.

Atrial fibrillation ablation is a complex procedure, because it involves a series of premises: 1 ) the patient needs to be in sinus rhythm with extrasystoles frequent enough to enable mapping of pulmonary veins, 2) transseptal puncture is necessary, 3) evolvement to atrial fibrillation during the procedure makes it more difficult because it requires repeated electrical cardioversion, 4) the number of radiofrequency applications must be limited so that pulmonary vein stenosis can be avoided, 5) mapping needs to be precise so that, ideally, only 1 pulmonary vein is selected for the application of radiofrequency.

We observed that patients with recurrent atrial fibrillation were not ideal candidates for mapping of pulmonary veins due to the difficulties in cardioversion and in the maintenance of sinus rhythm for mapping of atrial extrasystoles. If these patients wanted to undergo the procedure, we suggested that after successful electrical cardioversion, 24hour Holter monitoring be performed. Patients who experienced frequent atrial ectopy were considered eligible. However, a greater chance existed in these patients that the procedure would be more difficult.

In the subgroup of patients with short episodes of apparent atrial fibrillation on 24-hour Holter monitoring (6 patients), the success rate was $66 \%$ ( 4 of 6 patients). These episodes of atrial fibrillation were actually episodes of non- sustained atrial tachycardia, allowing mapping of the focal source in the pulmonary veins. We believe that these patients are ideal candidates for mapping and ablation. Two patients (number 2 and 6) experienced more than 500 episodes of nonsustained atrial tachycardia simulating atrial fibrillation on 24-hour monitoring prior to the procedure. In these patients, mapping was made easier by the high density of the arrhythmia, resulting in success in both cases.

We did not observe complications due to the procedure. The main apprehension in applying radiofrequency in the interior or around the pulmonary veins is the risk of pulmonary vein stenosis, which may lead to pulmonary hypertension. This is the most common complication resulting from linear ablation (extensive application with the intent to form continuous lines) in the left atrium around pulmonary veins ${ }^{30}$. However, pulmonary vein stenosis has been described in the focal ablation of atrial fibrillation ${ }^{31,32}$. In these studies ${ }^{31}$, ablation was performed in more than 1 pulmonary vein with multiple procedures, or electrical isolation of the pulmonary vein was attempted ${ }^{32}$. In our series, we performed only 1 procedure in all patients, and we did not make radiofrequency applications in more than 1 pulmonary vein. We took special care during the radiofrequency application (applications lasted only $15 \mathrm{~s}$, and pain was used as a criterion for interrupting the application). Perhaps the absence of complications in our series is due to this set of factors.

Currently, indications for mapping and attempting ablation of atrial fibrillation are not yet definitely established. This is a field of intensive research and constant change. The results of our study, even though preliminary, seem to indicate that mapping and attempting ablation of atrial fibrillation are feasible and may be attempted in all patients with paroxysmal atrial fibrillation, without structural heart disease and with short episodes of nonsustained atrial fibrillation on 24-hour Holter monitoring.

Our series of patients is limited and perhaps a larger group of patients might enable better selection of candidates for mapping. Systematic application of a greater number of provocative maneuvers to induce atrial ectopy would probably allow more efficient mapping in some patients. Maneuvers used in this study were: isoproterenol infusion, atrial stimulation, and adenosine infusion. We did not use the Valsava maneuver or others mentioned in the literature, such as carotid massage and volume infusion, digoxin or verapamil. Of these, only the Valsalva maneuver has made significant alterations in the frequency of ectopies ${ }^{28}$.

In conclusion, according to our analysis, patients who have short episodes of atrial fibrillation on 24-hour Holter monitoring were the best candidates for mapping and ablation, with a final success rate of $66 \%$ in this group of patients (4 of 6 patients) and, based on our limited experience, patients with recurrent atrial fibrillation are not eligible for focal ablation. 


\section{References}

1. Bialy D, Lehman MH, SchumacherDN, et al. Hospitalization for arrhythmias in the United States: Importance of atrial fibrillation. J Am Coll Cardiol 1992: 19-41.

2. Prystowsky EN, Benson DW Jr, Fuster V, et al. Management of patients with atrial fibrillation: a statement for healthcare professionals from the Committee on Electrocardiography and Electrophysiology, American Heart Association. Circulation 1996; 93: 1262-77.

3. Atrial fibrillation investigators. Risk factors for stroke and efficacy of antithrombotic therapy in atrial fibrillation: analysis of a pooled data from five randomized controlled trials. Arch Intern Med 1994: 154: 1449-57.

4. Coplen SE, Antman EM, Berlin JA, Hewitt P, Chalmers TC. Efficacy and safety of quinidine therapy for maintenance of sinus rhythm after cardioversion. A metaanalysis of randomized control trials. Circulation 1990; 82: 1106-16.

5. Juul Moller S, Edvardsson N, Rehnqvist Ahlberg N. Sotalol versus quinidine for the maintenance of sinus rhythm after direct current conversion of atrial fibrillation. Circulation 1990; 82: 1932-9.

6. Crijins HJ, Van Gelder IC, Van Gilst WH, Hillege H, Gosselink ATM, Lie KI. Serial antiarrhythmic drug treatment to maintain sinus rythm after electrical cardioversion for chronic atrial fibrillation or atrial flutter. Am J Cardiol 1991; 68: 335-41

7. Gosselink ATM, Crijins HJ, Van Gelder IC, Hillege H, Wiesfeld AC, Lie KI. Lowdose amiodarone for maintenance of sinus rhythm after cardioversion of atrial fibrillation or flutter. JAMA 1992; 267: 3289-93.

8. Jackman WM, Wang X, Friday KJ, et al. Catheter ablation of acessory atrioventricular pathways (Wolff-Parkinson-White syndrome) by radiofrequency current. N Engl J Med 1991; 324: 1605-11.

9. Calkins H, Souza J,El-Atassi R, et al. Diagnosis and cure of the Wolff-ParkinsonWhite syndrome or paroxysmal supraventricular tachycardia during a single electrophysiologic test. N Engl J Med 1991; 324: 1612-7.

10. Lesh MD, Hare GFV, Schamp DJ, et al. Curative percutaneous catheter ablation using radiofrequency energy for acessory pathways in all locations: Results in 100 consecutive patients. J Am Coll Cardiol 1992; 19: 1203-9.

11. Cox JL, Boineau JP, Schuessler RB, et al. Successful surgical treatment of atrial fibrillation. Review and clinical update. JAMA 1991; 266: 1976-80.

12. Jaïs $P$, Haïssaguere $M, S h a h D C$, et al. A focal source of atrial fibrillation treated by discrete radiofrequency ablation. Circulation 1997; 95: 572-6.

13. Swartz JF, Pellersels G, Silvers J, Patten L, CervantezD. A catheter-based curative approach to atrial fibrillation in humans. Circulation 1994; 90(pt 2): I-335.

14. Pappone C, Oreto G, Lamberti F, et al. Catheter ablation of paroxysmal atrial fibrillation using a 3D mapping system. Circulation 1999; 100: 1203-8.

15. Waspe L, Brodman R, Kim SG. Susceptibility to atrial fibrillation and ventricular tachyarrhythmias in the Wolff-Parkinson-White syndrome: role of the accessory pathway. Am Heart J 1986; 112: 1141-52.
16. Hamer ME, Wilkinson WE, Clair WK, Page RL, McCarthy EA, Pritchett ELC. Incidence of symptomatic atrial fibrillation in patients with paroxysmal supraventricular tachycardia. J Am Coll Cardiol 1995; 25: 984-8.

17. Delise P, Gianfranchi L, Paparella N, et al. Clinical usefulness of slow pathway ablation in patients with both atrioventricular node reentrant tachycardia and atrial fibrillation. Eur Heart J 1996; 17(suppl): 271.

18. Haïssaguerre M, Marcus FI, Fischer B, et al. Radiofrequency catheter ablation in unusual mechanisms of atrial fibrillation: Report of three cases. J Cardiovasc Electrophysiology 1994; 5: 743-51.

19. Haïssaguerre $M$, Jaïs $P$, Shah DC, et al. Spontaneous initiation of atrial fibrillation by ectopic beats originating in the pulmonary veins. NEngl J Med 1998; 339: 659-66.

20. Arentz T, Ott P, Stockinger J, Blum T, Rosenthal JV, Kalusche D. Radiofrequency catheter ablation of focal atrial fibrillation. PACE 1998; 21(II): 963.

21. Konings KTS, Kirschhof CJHJ, Smeets JRLM, Wellens HJJ, Penn O, Alessie MA. High-density mapping of electrically induced atrial fibrillation in humans. Circulation 1994; 89: 1665-80.

22. Ortiz J, Niwano S, Abe H, Rudy Y, Jonhson NJ, Waldo AL. Mapping the conversion fo atrial flutter to atrial fibrillation and atial fibrillation to atrial flutter: Insights into mechanism. Circ Res 1994; 74: 882-94.

23. Haïssaguerre M, Shah DC, Jaïs P, Clémenty J. Role of catheter ablation for atrial fibrillation. Curr Opinion Cardiology 1997; 12: 18-23.

24. Lau CP, Tse HF, Ayers GM: Radiofrequency ablation of focal atrial fibrillation as guided by site of spontaneous reinitiation of atrial fibrillation after defibrillation. PACE 1998; 21(II): 922

25. ToddDM, Hobbs WJC, Fynn SP, Armstrong K, Garratt CJ. How common is focal atrial fibrillation in patients undergoing cardioversion? PACE 1999; 22(II): 792.

26. Takahashi A, Jaïs $P$, Shah D, et al. Successful focal ablation of ectopy reinitiating persistent atrial fibrillation. PACE 1999; 22(II): 905.

27. Vergara I, Martin D, Bahnson T, Sorrentino RA, Greenfield RA, Wharton JM. Clinical predictors of successful focal atrial fibrillation ablation. PACE 1999; 22(II): 772 .

28. Shah DC, Haïssaguerre M, Jaïs P, Takahashi A, Hocini M, Clémenty J. Provocative manoeuvers for inducing pulmonary vein ectopy. PACE 1999; 22(II): 738.

29. Haïssaguerre M, Jaïs P, Shah DC, et al. Plurifocal sources of atrial fibrillation initiation from the pulmonary veins. PACE 1999; 22(II): 707.

30. Robbins IM, Colvin EV, Doyle TP, et al. Pulmonary vein stenosis after catheter ablation of atrial fibrillation. Circulation 1998; 98: 1769-75.

31. Jaïs $P$, Shah $D$, Haïssaguerre $M$, et al. Pulmonary vein patency after radiofrequency ablation. PACE 1999; 22: 738 .

32. Scanavacca M, Sosa E, D’Ávila A, et al. Ablação com radiofrequiência da fibrilação atrial paroxística. Arq Bras Cardiol 1999; 72: 693-700. 